80262, USA. Phone: (303) 315-4475; Fax: (303) 315-5632; E-mail: Rubin.Tuder@ uchsc.edu.

1. Croxton, T.L., Weinmann, G.G., Senior, R.M. and Hoidal, J.R. 2002. Future research directions in chronic obstructive pulmonary disease. Am. J. Respir. Crit. Care Med. 165:838-844.

2. Tuder, R.M., Yoshida, T., Arap, W., Pasqualini, R., and Petrache, I. 2006. Cellular and molecular mechanisms of alveolar destruction in emphysema: an evolutionary perspective. Proc. Am. Thorac. Soc. 3:503-510.

3. Tuder, R.M., Yun, J.H., and Graham, B.B. 2008. Cigarette smoke triggers code red:p21CIP1/WAF1/ SDI1 switches on danger responses in the lung. Am. J. Respir. Cell Mol. Biol. 39:1-6.

4. Balaban, R.S., Nemoto, S., and Finkel, T. 2005. Mitochondria, oxidants, and aging. Cell. 120:483-495.

5. Kang, M.-J., et al. 2008. Cigarette smoke selectively enhances viral PAMP- and virus-induced pulmonary innate immune and remodeling responses in mice. J. Clin. Invest. 118:2771-2784.

6. Tuder, R.M., Petrache, I., Elias, J.A., Voelkel, N.F., and Henson, P.M. 2003. Apoptosis and emphysema: the missing link. Am. J. Respir. Cell Mol. Biol. 28:551-554.

7. Papi, A., et al. 2006. Infections and airway inflammation in chronic obstructive pulmonary disease severe exacerbations. Am. J. Respir. Crit. Care Med. 173:1114-1121.

8. Akira, S., Uematsu, S., and Takeuchi, O. 2006. Pathogen recognition and innate immunity. Cell. 124:783-801.

9. Kawai, T., and Akira, S. 2006. Innate immune recognition of viral infection. Nat. Immunol. 7:131-137.

10. Garcia, M.A., et al. 2006. Impact of protein kinase PKR in cell biology: from antiviral to antiproliferative action. Microbiol. Mol. Biol. Rev. 70:1032-1060.

11. Sun, Q., et al. 2006. The specific and essential role of MAVS in antiviral innate immune responses. Immunity. 24:633-642.

12. Seth, R.B., Sun, L., and Chen, Z.J. 2006. Antiviral innate immunity pathways. Cell Res. 16:141-147.

13. Der, S.D., Yang, Y.L., Weissmann, C., and Williams, B.R. 1997. A double-stranded RNA-activated protein kinase-dependent pathway mediating stressinduced apoptosis. Proc. Natl. Acad. Sci. U. S. A. 94:3279-3283.
14. Zheng, T., et al. 2005. Role of cathepsin S-dependent epithelial cell apoptosis in IFN- $\gamma$-induced alveolar remodeling and pulmonary emphysema. J. Immunol. 174:8106-8115.

15. Grath-Morrow, S., et al. 2008. Impaired lung homeostasis in neonatal mice exposed to cigarette smoke. Am. J. Respir. Cell Mol. Biol. 38:393-400.

16. Yoshida, T., and Tuder, R.M. 2007. Pathobiology of cigarette smoke-induced chronic obstructive pulmonary disease. Physiol. Rev. 87:1047-1082.

17. Rangasamy, T., et al. 2004. Genetic ablation of Nrf2 enhances susceptibility to cigarette smoke-induced emphysema in mice. J. Clin. Invest. 114:1248-1259.

18. Sato, T., et al. 2006. Senescence marker protein30 protects mice lungs from oxidative stress, aging, and smoking. Am. J. Respir. Crit. Care Med. 174:530-537.

19. Elias, J.A., Kang, M.J., Crouthers, K., Homer, R., and Lee, C.G. 2006. State of the art. Mechanistic heterogeneity in chronic obstructive pulmonary disease: insights from transgenic mice. Proc. Am. Thorac. Soc. 3:494-498.

20. Taraseviciene-Stewart, L., and Voelkel, N.F. 2008 Molecular pathogenesis of emphysema. J. Clin. Invest. 118:394-402

\title{
Lipid droplets: FSP27 knockout enhances their sizzle
}

\author{
Vishwajeet Puri and Michael P. Czech \\ Program in Molecular Medicine, University of Massachusetts Medical School, Worcester, Massachusetts, USA.
}

\begin{abstract}
Fat-specific protein of $27 \mathrm{kDa}$ (FSP27) is a highly expressed adipocyte protein that promotes triglyceride accumulation within lipid droplets. In this issue of the JCI, Nishino et al. show that FSP27 also helps to maintain the characteristically large unilocular lipid droplet structure within each white adipocyte (see the related article beginning on page 2808). Fragmentation of lipid droplets in white adipocytes from FSP27-KO mice caused both increased lipolysis and upregulation of genes enhancing mitochondrial oxidative metabolism. This increased energy expenditure in turn protected the mice from diet-induced obesity and insulin resistance. These new results highlight powerful mechanisms that tightly coordinate rates of triglyceride storage in lipid droplets with mitochondrial fatty acid oxidation in white adipocytes.
\end{abstract}

The handling and disposal of large volumes of greasy cooking ingredients like lard and vegetable oils is a messy business as any restaurant manager will lament. Human physiology faces the same daunting challenge for the efficient storage and metabolism of fats. To accomplish these feats, fat-storing cells, referred to as white

Nonstandard abbreviations used: CIDE, cell deathinducing DNA fragmentation factor 45-like effector; FSP27, fat-specific protein of $27 \mathrm{kDa}$; WAT, white adipose tissue.

Conflict of interest: The authors have declared that no conflict of interest exists.

Citation for this article: J. Clin. Invest. 118:2693-2696 (2008). doi:10.1172/JCI36554. fat cells or adipocytes, are uniquely specialized to accommodate over $95 \%$ of their cell volume as a single lipid droplet (Figure 1). Triglyceride within this unilocular lipid droplet can, in reaction to other signals, be hydrolyzed to fatty acids and glycerol, which move through the circulation to muscle and other tissues for energy production (fatty acids) and to liver for conversion to glucose (glycerol). Proteins are known to coat the surfaces of lipid droplets in adipocytes. However, until now, what molecules are required to maintain the distinctive unilocular characteristic of lipid droplets in white adipocytes has remained a mystery. In this issue of the JCI, Nishino et al.
(1) provide compelling evidence that one such protein is fat-specific protein of 27 $\mathrm{kDa}$ (FSP27; also known as Cidec), a highly and selectively expressed protein in human white adipocytes (2), recently found to colocalize with lipid droplets and regulate fat storage (3-5).

\section{Lipid droplets: composition and structure}

Lipid droplets within adipocytes are composed of a core of triglycerides and cholesteryl esters that are surrounded on their surfaces by a phospholipid monolayer associated with lipid droplet proteins (6). Perilipin was the first identified lipid droplet-specific protein that coats the surfaces of these structures in brown and white adipocytes and some steroidogenic cells (7). It is the prototypic member of a family of related proteins containing a "PAT" domain, so called for the members perilipin, a dipophilin (also known as adipose differentiation-related protein), and the tail-interacting protein of $47 \mathrm{kDa}$ (TIP47), but also includes the plasma membrane-associated protein S3-12 and myocardial lipid droplet protein/oxidative tissues-enriched PAT protein (MLDP/OXPAT) $(6,8)$. This domain, in 


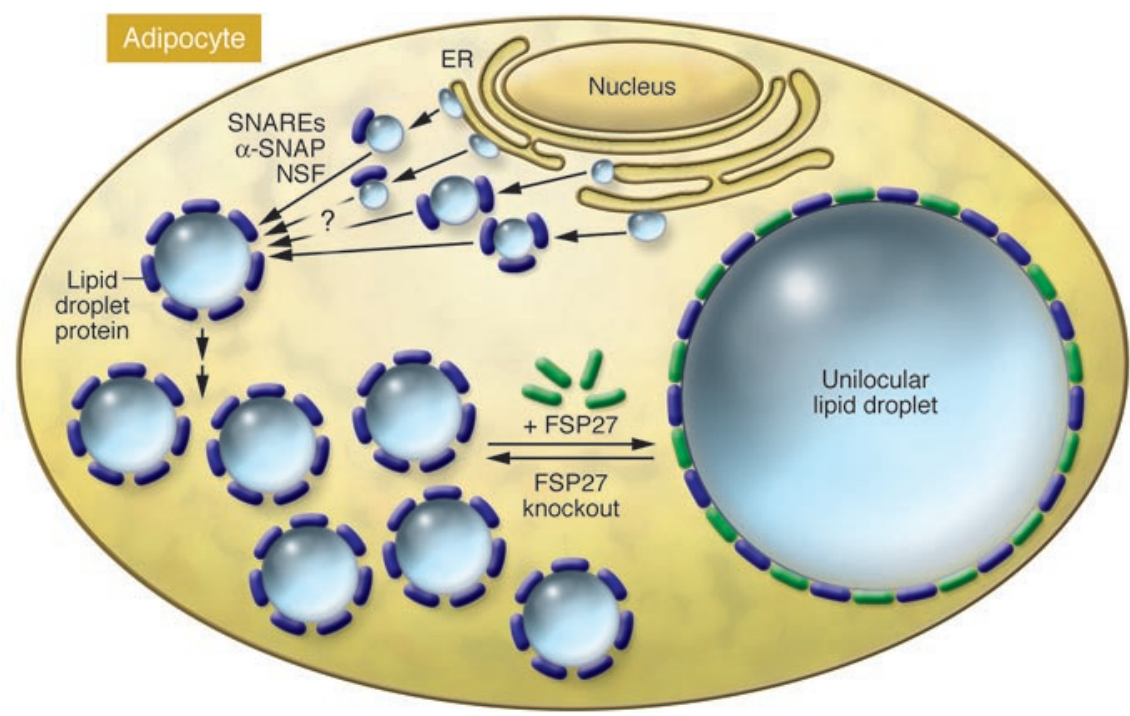

Figure 1

FSP27 is required for the unilocular structure of lipid droplets in white adipocytes. According to this model, the biogenesis of small lipid droplets in the endoplasmic reticulum is followed by their subsequent fusion in the cytoplasm of white adipocytes. Proteins thought to be involved in cell membrane fusion processes (e.g., N-ethylmaleimide-sensitive factor [NSF], soluble NSF attachment protein [ $\alpha$-SNAP], and SNAP receptors [SNAREs]) might activate these fusion processes. Lipid droplet proteins (dark blue) such as perilipin, tail-interacting protein of $47 \mathrm{kDa}$, S3-12, adipose differentiation-related protein, and others may be involved in packaging the lipids in multilocular lipid droplets in adipocytes. According to Nishino et al. (1), in their current study in this issue of the $\mathrm{JCl}$, FSP27 plays a key role in the formation of unilocular lipid droplets in adipocytes of WAT, since lipid droplets are shown to be multilocular in FSP27-KO mice.

combination with others, functions to target these proteins to lipid droplets and to mediate regulation of triglyceride storage and release from the droplets. Within the past year, additional proteins were discovered to be colocalized with cellular lipid droplets, including FSP27 (3, 4), Cidea (9), and fatinducing transcript 1 (FIT1) and FIT2, endoplasmic reticulum-resident membrane proteins that induce lipid droplet accumulation in cell culture and when expressed in mouse liver (10). FSP27 and Cidea are members of the cell death-inducing DNA fragmentation factor 45-like effector (CIDE) protein family, members of which share a conserved $\mathrm{N}$-terminal CIDE-N domain and a $\mathrm{COOH}$ terminal CIDE-C domain $(3,5)$. When expressed in preadipocytes or even African green monkey kidney cells (COS cells), these proteins enhance the deposition of triglyceride and the size of lipid droplets $(3,5)$. Three isoforms of the CIDE proteins have been reported in mice (Cidea, Cideb, and FSP27) and humans (CIDEA, CIDEB, and CIDEC). Human CIDEC is the homolog of mouse FSP27. Interestingly, like FSP27 and Cidea, Cideb might also be a hepatic lipid droplet regulator, since its ablation in mice causes decreased fat accumulation in liver (11).
How might FSP27 mediate unilocular disposition of the lipid droplet in primary white adipocytes? This function of FSP27 is not apparently shared by perilipin, because in their current study, Nishino et al. (1) show that white adipocytes from FSP27$\mathrm{KO}$ mice are multilocular, while those from perilipin-KO mice remain unilocular $(12,13)$. Little is known about the mechanisms that control the sizes of lipid droplets in adipocytes or in other cells that can accumulate small lipid droplets. However, proteins involved in cell membrane fusion processes, such as N-ethylmaleimide-sensitive factor (NSF), soluble NSF attachment protein ( $\alpha$-SNAP), and such SNAP receptors (SNAREs) as synaptosomal-associated protein of $23 \mathrm{kDa}$ (SNAP23), syntaxin-5, and vesicle-associated membrane protein 4 (VAMP4), were recently discovered to be associated with lipid droplets and may mediate lipid droplet fusion (14). Thus, as depicted in Figure 1, perhaps the biogenesis of small lipid droplets in the endoplasmic reticulum is followed by their subsequent fusion in the cytoplasm of adipocytes. Continued enlargement of lipid droplets by direct incorporation of additional triglycerides might also occur.
In this regard, some lipid droplet proteins are constitutively available in the cytoplasm to bind lipid droplets (e.g., TIP47, S3-12, MLDP/OXPAT), while others are exclusively present on lipid droplets and absent as free proteins in the cytoplasm (e.g., perilipin, Cidea, and FSP27). As the model in Figure 1 hypothesizes, perhaps FSP27 catalyzes fusions of smaller lipid droplets onto the larger one, although this concept remains to be rigorously tested. Interestingly, Nishino et al. show that the presence of multilocular lipid droplets in brown adipocytes correlates with virtually undetectable levels of FSP27 protein in these cells. In contrast, perilipin is abundant in brown adipocytes.

\section{Perilipin and FSP27 in control of lipolysis and fat storage}

Another important distinction between the functions of perilipin and FSP27 revealed by the current study by Nishino et al. (1), relates to their modes of regulation of lipolysis. Perilipin is required for optimal glycerol and fatty acid release into the serum in response to injection of catecholamines into mice (12), while FSP27 ablation has no effect on this response (1). Thus, perilipin is thought to be part of the mechanism whereby lipolysis is stimulated through enhancement of the actions of adipose triglyceride lipase (ATGL) (6) and hormone-sensitive lipase $(6,8,15)$, while FSP27 does not seem to function directly in these mechanisms. However, in cultured adipocytes, siRNA-mediated knockdown of either FSP27 or perilipin enhances basal lipolysis, without attenuating lipolysis in response to catecholamines $(1,3)$. This difference between cultured versus primary adipocytes with respect to the mode by which perilipin participates in catecholamine action is not understood and contrasts with the similarity of effects of FSP27 in vivo and in vitro. In any case, FSP27 depletion by either gene ablation in mice (1) or by siRNA-mediated gene silencing in cultured adipocytes (3) causes marked fragmentation of lipid droplets, which may stimulate lipolysis through enhanced lipase action on the larger surface area per unit volume of triglyceride. Importantly, Nishino et al. show that cells depleted of both perilipin and FSP27 yielded no further lipid droplet fragmentation than cells depleted of FSP27 alone, yet lipolysis was increased further in the case of the former (1). Taken together, these findings support the idea that different mechanisms of 


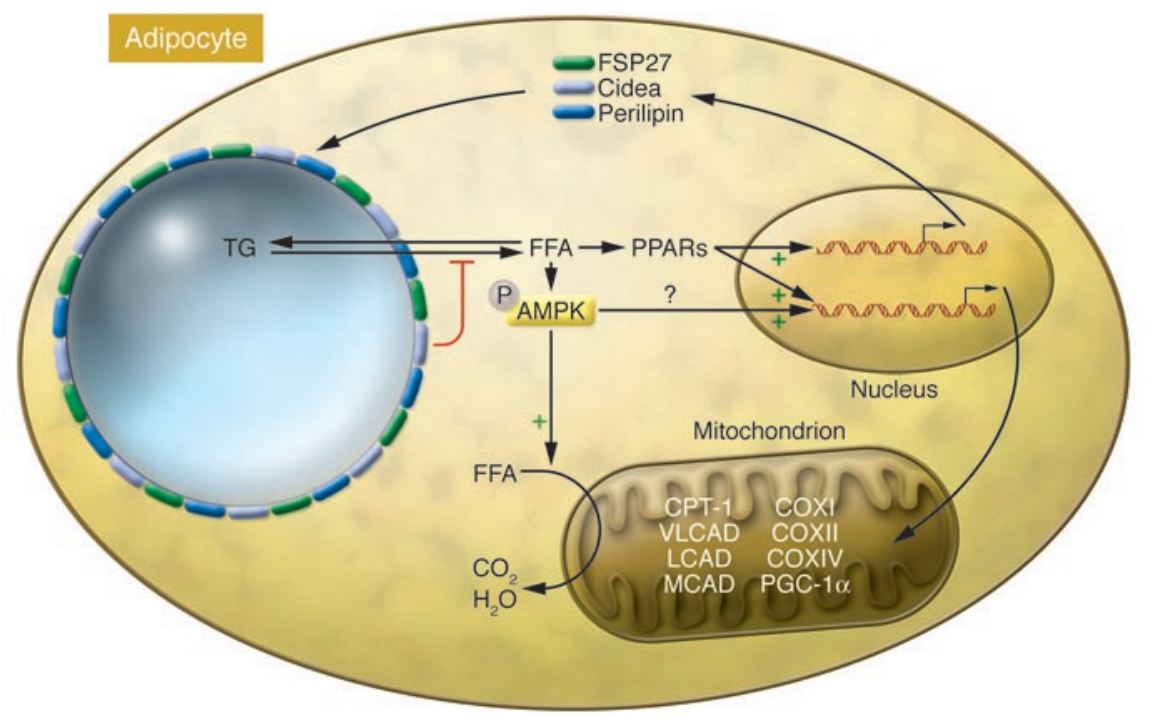

Figure 2

FFA-induced gene expression and oxidative metabolism in FSP27-KO mice. The lipid droplet proteins FSP27, Cidea, and perilipin are regulated by PPAR $\gamma$ and play a role in lipid droplet biogenesis and dynamics. These lipid droplet proteins inhibit lipolysis (red inhibitory symbol). Thus, depletion of these lipid droplet proteins enhances adipocyte lipolysis, releasing glycerol and FFAs. According to this model, FFAs released during lipolysis induced by depletion of FSP27, Cidea, or perilipin may act as ligands for PPARs that further regulate the transcriptional coactivator PGC-1 $\alpha$ as well as genes that encode proteins that promote mitochondrial biogenesis and fatty acid oxidation. Similarly, FFAs released during lipolysis have been shown to activate AMPK, which functions to stimulate fatty acid oxidation, by increasing fatty acid transport into mitochondria and perhaps by regulating the transcription of genes that encode proteins that promote fatty acid oxidation. Thus, the increased mitochondrial oxidation of fatty acids in white adipocytes of FSP27-KO mice may result in part from activation of AMPK. In addition, fragmentation of lipid droplets in FSP27-KO mice may enhance access of mitochondria to the resulting increased surface area of lipid droplets, further augmenting fatty acid oxidation (data not shown, see text for details). COXI, subunit I of cytochrome $c$ oxidase; CPT-1, carnitine palmitoyltransferase-1; LCAD, long-chain acyl-coenzyme A dehydrogenase; MCAD, medium-chain acyl-coenzyme A dehydrogenase; PGC-1 $\alpha$, PPAR $\gamma$-coactivator $1 \alpha$; TG, triglyceride; VLCAD, very LCAD.

action characterize perilipin versus FSP27, with respect to regulation of lipolysis in primary white adipocytes.

\section{Lipid droplets and whole-body glucose homeostasis}

The results obtained using the FSP27-KO mice generated by Nishino et al. (1) reveal a fascinating and instructive paradox when compared with effects of perilipin loss on whole-body metabolism. In both perilipin$\mathrm{KO}$ and FSP27-KO animals, the amount of white adipose tissue (WAT) is markedly reduced $(1,12,13)$. Similarly, both FSP27and perilipin-KO mice show increased oxygen consumption and energy expenditure compared with wild-type mice, in concert with increased availability of fatty acid substrate emanating from unshielded lipid droplets. Yet, despite these similarities, Nishino et al. show FSP27-KO mice remain consistently insulin-sensitive, even remains. Importantly, mitochondria localize around lipid droplets (17), which makes fatty acid substrates highly accessible. Thus, the increased surface area on the many small lipid droplets in FSP27-KO adipocytes likely enhances mitochondrial access to fatty acid substrates. Together, these effects may greatly increase fatty acid oxidation within WAT in the FSP27-depleted animal and attenuate the release of fatty acids to muscle and liver where they can cause insulin resistance. Increased release of fatty acids in the perilipin-KO mice may cause increased fatty acids to enter skeletal muscle and liver. Fatty acid oxidation is indeed enhanced in these tissues in the perilipin-KO mouse but not in the FSP27-KO animals. Thus, the unique lipid droplet fragmentation in WAT of the FSP27-KO mouse may uniquely elevate the fatty acid oxidation capacity of the WAT itself. This leads to a stunning, positive influence on whole-body glucose homeostasis. This concept is consistent with previous results in which the uncoupling protein UCP1 was expressed in WAT to increase its fatty acid oxidizing capacity, which enhanced whole-body glucose tolerance (18). Interestingly, part of this effect may be mediated through neuronal circuits between WAT and the brain (18).

\section{Mechanisms linking lipid droplets to mitochondrial energetics}

How might lipid droplet fragmentation and increased levels of intracellular fatty acids due to FSP27 ablation augment mitochondrial biogenesis and their capacity for fatty acid oxidation? Two candidates present themselves as likely intermediaries of these effects of increased intracellular fatty acids on mitochondrial function (Figure 2). The first includes nuclear receptors such as the PPAR protein family, known to be responsive to fatty acids and their derivatives. PPAR $\gamma$ powerfully promotes adipogenesis, a process also associated with increased mitochondrial biogenesis (17). PPAR $\gamma$ agonists such as the thiazolidinediones further augment expression of genes related to fatty acid oxidation and oxidative phosphorylation in mature, fully differentiated adipocytes in culture (17). It is also well established that PPAR $\gamma$ isoforms PPAR $\alpha$ and PPAR $\delta$ expressed in WAT strongly promote expression of genes in mitochondrial oxidative pathways (19-21). These latter transcription factors are most highly expressed in skeletal muscle (PPAR $\delta$ ) and liver (PPAR $\alpha$ ) and could be important intermediaries of the increased fatty acid 
oxidation observed in these tissues in the perilipin-KO mouse. A key experiment to test whether PPAR isoforms contribute to increased mitochondrial function in response to FSP27 depletion would be to perform double knockdowns in fully mature adipocytes.

A second potential intermediate between FSP27 and mitochondrial oxidative metabolism could be the protein kinase AMPK (Figure 2), which is a key regulator of fatty acid oxidation in response to increased intracellular AMP levels (reviewed in ref. 22). The AMP/ATP ratio in isolated adipocytes is indeed increased under lipolytic conditions, and this is attenuated when fatty acid levels are reduced (23). Elevated intracellular free fatty acids in adipocytes might increase AMP/ATP ratios through uncoupling effects in mitochondria or by enhancing ATP utilization for fatty acid acylation by coenzyme A (23). Previous studies implicate AMPK in mediating increased transcription of adipocyte genes in the fatty acid oxidation pathway (24), although further work is required to substantiate this mode of regulation and unravel the targets of AMPK signaling in this pathway. Nonetheless, the idea that AMPK acts in conjunction with the lipid droplet protein FSP27 to finetune the relationship between fatty acid esterification versus fatty acid oxidation in mitochondria is a worthy hypothesis for vigorous investigation (Figure 2). Similarly, PPAR $\gamma$ may act to promote mitochondrial fatty acid oxidation along with fatty acid acylation and esterification because the latter processes require the ATP generated by the former process.

\section{A role for FSP27 in human obesity?}

In normal humans, expression of perilipin and FSP27 mRNA appears to be over 100 times higher in adipose tissue than in dozens of other tissues studied (25). Cidea is also present in human adipose tissues, and its expression inversely correlates with insulin resistance in obese human subjects $(9,26)$. Moreover, a polymorphism in Cidea correlates with the incidence of obesity in humans $(27,28)$. Very recently, we reported that omental adipose tissue samples derived from obese human subjects with similar BMI values exhibited levels of perilipin, FSP27, and CIDEA mRNA that also inversely correlate with the extent of insulin resistance (9). However, the lipid droplets in adipocytes from all subjects remained unilocular, consistent with the fact that substantial levels of FSP27 remained. Thus, increased WAT fatty acid oxidation may not be greatly stimulated under these conditions. Taken together, these results converge with the generally held idea that insulin resistance is mediated in part by the inability of adipocytes to sequester triglyceride away from muscle and liver. It is plausible that deficiencies in lipid droplet proteins that promote lipid storage, including FSP27, compromise WAT fat sequestration and contribute to increased circulating fatty acids that promote insulin resistance in skeletal muscle and liver. Nishino et al. (1) have provided insightful data that may be important in further testing such hypotheses.

\section{Acknowledgments}

The authors gratefully acknowledge their lab colleagues for helpful discussions. We unfortunately could not cite all relevant publications due to the limited citations allowed for this article. The work described from our laboratory was supported by National Institutes of Health grants DK30898 and DK60837 to M.P. Czech.

Address correspondence to: Michael P. Czech, Program in Molecular Medicine, University of Massachusetts Medical School, 373 Plantation Street, Worcester, Massachusetts 01605, USA. Phone: (508) 856-2254; Fax: (508) 856-1617; E-mail: michael.czech@umassmed.edu.

1. Nishino, N., et al. 2008. FSP27 contributes to efficient energy storage in murine white adipocytes by promoting the formation of unilocular lipid droplets. J. Clin. Invest. 118:2808-2821.

2. Danesch, U., Hoeck, W., and Ringold, G.M. 1992. Cloning and transcriptional regulation of a novel adipocyte-specific gene, FSP27. CAAT-enhancerbinding protein $(\mathrm{C} / \mathrm{EBP})$ and $\mathrm{C} / \mathrm{EBP}-\mathrm{like}$ proteins interact with sequences required for differentiationdependent expression. J. Biol. Chem. 267:7185-7193.

3. Puri, V., et al. 2007. Fat-specific protein 27 , a novel lipid droplet protein that enhances triglyceride storage. J. Biol. Chem. 282:34213-34218.

4. Puri, V., Virbasius, J.V., Guilherme, A., and Czech, M.P. 2008. RNAi screens reveal novel metabolic regulators: RIP140, MAP4k4 and the lipid droplet associated fat specific protein (FSP) 27. Acta Physiol. (Oxf.). 192:103-115.

5. Keller, P., et al. 2008. Fat-specific protein 27 regulates storage of triacylglycerol. J. Biol. Chem. 283: $14355-14365$.

6. Brasaemle, D.L. 2007. Thematic review series: adipocyte biology. The perilipin family of structural lipid droplet proteins: stabilization of lipid droplets and control of lipolysis. J. Lipid Res. 48:2547-2559.

7. Londos, C., et al. 1995. Perilipin: unique proteins associated with intracellular neutral lipid droplets in adipocytes and steroidogenic cells. Biochem. Soc. Trans. 23:611-615.

8. Ducharme, N.A., and Bickel, P.E. 2008. Lipid droplets in lipogenesis and lipolysis. Endocrinology. 149:942-949.

9. Puri, V., et al. 2008. Cidea is associated with lipid droplets and insulin sensitivity in humans. Proc. Natl. Acad. Sci. U. S. A. 105:7833-7838.

10. Kadereit, B., et al. 2008. Evolutionarily conserved gene family important for fat storage. Proc. Natl. Acad. Sci. U. S. A. 105:94-99.

11. Matsusue, K., et al. 2008. Hepatic steatosis in leptin-deficient mice is promoted by the PPARgamma target gene Fsp27. Cell Metab. 7:302-311.

12. Martinez-Botas, J., et al. 2000. Absence of perilipin results in leanness and reverses obesity in Lepr $(\mathrm{db} / \mathrm{db})$ mice. Nat. Genet. 26:474-479.

13. Tansey, J.T., et al. 2001. Perilipin ablation results in a lean mouse with aberrant adipocyte lipolysis, enhanced leptin production, and resistance to diet-induced obesity. Proc. Natl. Acad. Sci. U. S. A. 98:6494-6499.

14. Bostrom, P., et al. 2007. SNARE proteins mediate fusion between cytosolic lipid droplets and are implicated in insulin sensitivity. Nat. Cell Biol. 9:1286-1293.

15. Tansey, J.T., Sztalryd, C., Hlavin, E.M., Kimmel, A.R., and Londos, C. 2004. The central role of perilipin a in lipid metabolism and adipocyte lipolysis. IUBMB Life. 56:379-385.

16. Saha, P.K., Kojima, H., Martinez-Botas, J., Sunehag, A.L., and Chan, L. 2004. Metabolic adaptations in the absence of perilipin: increased beta-oxidation and decreased hepatic glucose production associated with peripheral insulin resistance but normal glucose tolerance in perilipin-null mice. J. Biol. Chem. 279:35150-35158.

17. Wilson-Fritch, L., et al. 2004. Mitochondrial remodeling in adipose tissue associated with obesity and treatment with rosiglitazone. J. Clin. Invest. 114:1281-1289.

18. Yamada, T., et al. 2006. Signals from intra-abdominal fat modulate insulin and leptin sensitivity through different mechanisms: neuronal involvement in food-intake regulation. Cell Metab. 3:223-229.

19. Villarroya, F., Iglesias, R., and Giralt, M. 2007. PPARs in the control of uncoupling proteins gene expression. PPAR Res. 2007:74364.

20. Bogacka, I., Ukropcova, B., McNeil, M., Gimble, J.M., and Smith, S.R. 2005. Structural and functional consequences of mitochondrial biogenesis in human adipocytes in vitro. J. Clin. Endocrinol. Metab. 90:6650-6656.

21. Forman, B.M., Chen, J., and Evans, R.M. 1997. Hypolipidemic drugs, polyunsaturated fatty acids, and eicosanoids are ligands for peroxisome proliferator-activated receptors alpha and delta. Proc. Natl. Acad. Sci. U. S. A. 94:4312-4317.

22. Long, Y.C., and Zierath, J.R. 2006. AMP-activated protein kinase signaling in metabolic regulation. J. Clin. Invest. 116:1776-1783.

23. Gauthier, M.S., et al. 2008. AMP-activated protein kinase is activated as a consequence of lipolysis in the adipocyte: potential mechanism and physiological relevance. J. Biol. Chem. 283:16514-16524.

24. Leff, T. 2003. AMP-activated protein kinase regulates gene expression by direct phosphorylation of nuclear proteins. Biochem. Soc. Trans. 31:224-227.

25. Su, A.I., et al. 2002. Large-scale analysis of the human and mouse transcriptomes. Proc. Natl. Acad. Sci. U. S. A. 99:4465-4470.

26. Nordstrom, E.A., et al. 2005. A human-specific role of cell death-inducing DFFA (DNA fragmentation factor-alpha)-like effector A (CIDEA) in adipocyte lipolysis and obesity. Diabetes. 54:1726-1734.

27. Dahlman, I., et al. 2005. The CIDEA gene V115F polymorphism is associated with obesity in Swedish subjects. Diabetes. 54:3032-3034.

28. Zhang, L., Miyaki, K., Nakayama, T., and Muramatsu, M. 2008. Cell death-inducing DNA fragmentation factor alpha-like effector A (CIDEA) gene V115F (G-->T) polymorphism is associated with phenotypes of metabolic syndrome in Japanese men. Metabolism. 57:502-505. 